\title{
Antimicrobial resistance profile of Staphylococcus aureus isolated from patients with infection at Tikur Anbessa Specialized Hospital, Addis Ababa, Ethiopia
}

\author{
Sileshi Tadesse ${ }^{1}$, Haile Alemayehu ${ }^{2}$, Admasu Tenna ${ }^{3}$, Getachew Tadesse ${ }^{4}$, Tefaye Sisay Tessema ${ }^{5}$,
}

Workineh Shibeshi ${ }^{6}$ and Tadesse Eguale ${ }^{2^{*}}$ (D)

\begin{abstract}
Background: Staphylococcus aureus is one of the major pathogens of public health importance responsible for various forms of infection. Development of resistance to commonly used antimicrobials limited treatment options against infections due to this pathogen. Antimicrobial resistance profile of Staphylococcus aureus isolated from patients with surgical site infection and ear infection and corresponding nasal swab was investigated in Tikur Anbessa Specialized Hospital (TASH), Addis Ababa, Ethiopia.
\end{abstract}

Methods: Wound and corresponding nasal swabs from patients with surgical site infection from general surgery ward $(n=14)$, orthopedic ward $(n=21)$ and those with otitis media $(n=59)$ from Ear Nose and Throat (ENT) ward were cultured for S. aureus isolation according to standard procedures from December 2013 to June 2014. Isolates were investigated for susceptibility to panel of 17 antimicrobials using Kirby Bauer disc diffusion assay. Susceptibility to methicillin was phenotypically determined based on sensitivity of isolates to cefoxitin and oxacillin.

Results: A total of 79 S. aureus isolates were recovered from 54(57.4\%) of patients. The isolates were resistant to ampicillin (100\%), oxacillin and cefoxitin (68.4\%, each), clindamycin (63.3\%), cephalothin (59.5\%), tetracycline (57\%), sulfamethoxazole + trimethoprim and bacitracin (53.2\%, each), and erythromycin (51.9\%). Resistance to two or more antimicrobials was recorded in 74 (95\%) of the isolates, while resistance to 3 or more antimicrobials was detected in 65(82.3\%) of the isolates. Fifty-four (68.4\%) of the isolates were methicillin resistant S. aureus (MRSA). Rate of occurrence of MRSA was more common among isolates from surgical wards $(p<0.001)$ compared to those from ENT ward. High level of multi-drug resistance (MDR) was detected more commonly among isolates from orthopedic ward than those from general surgical ward and patients with ear infection $(p<0.001)$. One of the isolate cultured from wound swab of a patient with surgical site infection from orthopedic ward was resistant to all of the 17 antimicrobials tested.

Conclusion: S. aureus isolates from patients in TASH exhibited resistance to majority of antimicrobials commonly employed for the treatment of staphylococcal infections which calls for urgent need of prudent use of antimicrobials and the need for implementation of effective infection control practices to hamper spread of MDR S. aureus.

Keywords: Staphylococcus aureus, Methicillin resistant Staphylococcus aureus, Surgical site infection, Drug resistance, Otitis media

\footnotetext{
* Correspondence: tadesse.eguale@aau.edu.et

${ }^{2}$ Aklilu Lemma Institute of Pathobiology, Addis Ababa University, P.O. Box

1176, Addis Ababa, Ethiopia

Full list of author information is available at the end of the article
}

(c) The Author(s). 2018 Open Access This article is distributed under the terms of the Creative Commons Attribution 4.0 International License (http://creativecommons.org/licenses/by/4.0/), which permits unrestricted use, distribution, and reproduction in any medium, provided you give appropriate credit to the original author(s) and the source, provide a link to the Creative Commons license, and indicate if changes were made. The Creative Commons Public Domain Dedication waiver (http://creativecommons.org/publicdomain/zero/1.0/) applies to the data made available in this article, unless otherwise stated. 


\section{Background}

Staphylococcus aureus is a normal flora associated with skin, skin glands and mucous membrane of almost all warm blooded animals and about $30 \%$ of the human population is colonized by $S$. aureus [1]. It is also a leading cause of life- threatening blood stream infection, osteoarticular, skin, soft tissue, respiratory tract, deviceassociated and surgical site infections particularly in immunocompromised, young and elderly patients [2]. The ability of $S$. aureus to invade the host immune system through various virulence factors and its rapid acquisition of multi-drug resistance phenotype, makes it one of the most notorious organism among gram positive bacterial pathogens [3].

The burden of infection with antimicrobial resistant strains of pathogens involves increased risk of mortality, increased hospital stay, and related attributable costs compared to infection with antimicrobial susceptible pathogens [4]. S. aureus is reported to be the most common cause of nosocomial infections and is particularly responsible for majority of surgical site infections [5]. In a previous study in northern Ethiopia, the rate of surgical site infection accounted for $10.2 \%$ and $S$. aureus was shown to be the leading bacterial pathogen responsible for surgical site infection [6]. Similarly, S. aureus was the most frequently isolated pathogen among patients with otitis media at Bahir Dar, northwest Ethiopia [7], Ayder teaching and Referral Hospital, Mekelle, northern Ethiopia [8] and isolates were resistant to several antimicrobials.

Multidrug resistant (MDR) strains of $S$. aureus, particularly methicillin resistant Staphylococcus aureus (MRSA) have potential of rapid spread in a given health facility through colonized or infected patients or health personnel as well as contaminated environments in the facilities, unless there is strict infection control strategy [9]. Recent study showed that infection with MDR strains of $S$. aureus is associated with prolonged length of hospital stay and increased mortality [10]. MRSA is one of the major public health threats globally. Antimicrobial resistance in MRSA is associated with acquisition of large mobile genetic element called staphylococcal cassette chromosome (SCCmec) which carries the central determinant for a broad spectrum beta-lactam resistance encoded by mecA or $m e c C$ genes $[11,12]$. Studies showed widespread distribution of MRSA in various countries particularly in hospital environments [13-15] as well as occurrence of community and livestock associated MRSA [16-18]. In addition to $m e c \mathrm{~A}$ and $m e c \mathrm{C}$ genes, SCC was reported to carry several other drug resistance genes which confer resistance to mercury, kanamycin, erythromycin, spectinomycin and fusidic acid [19]. Moreover, recent studies showed dramatic increase in the development of resistance to vancomycin, the other alternative drug for the treatment of infections caused by gram positive organisms [20].
It has been shown that overall epidemiology, pathophysiology and clinical manifestations of S. aureus vary significantly among different countries and different regions of the same country [2]. In most developing countries like Ethiopia, the potential public health threat due to antimicrobial resistance is high because of the fact that antimicrobial agents can easily be purchased without prescription [21], lack of coordinated routine surveillance of antimicrobial resistance, poor laboratory capacity, and poor infection control mechanisms by health facilities [22]; contributing to the emergence and spread of antimicrobial resistance [23]. Knowledge on the antimicrobial susceptibility status of circulating pathogens in a given health facility is important for better management of infectious pathogens particularly where routine culture and sensitivity testing is not practiced. Recent study at Yekatit 12 Hospital, Addis Ababa, showed isolation rate of $S$. aureus from $14.3 \%$ of clinical specimens, of which over $50 \%$ of the isolates were MDR and $17.5 \%$ were MRSA [24]. Scant information is available on the occurrence and antimicrobial susceptibility of $S$. aureus in patients with surgical site infection and those with otitis media in Ethiopia. Thus, the present study reports occurrence and antimicrobial resistance profile of $S$. aureus among patients with surgical site infection and ear infection and corresponding nasal swab at Tikur Anbessa Specialized Hospital (TASH), Addis Ababa, Ethiopia.

\section{Methods}

\section{Study setting, study design and subjects}

The study was conducted at Tikur Anbessa Specialized Hospital, a tertiary teaching hospital of Addis Ababa University, from December 2013 to June, 2014. The hospital provides diverse services for patients from different parts of the country. The study design was a hospital based cross-sectional study in which hospitalized patients from general surgical ward and orthopedic ward as well as outpatients from ear, nose, and throat (ENT) ward were involved. Patients with clinical evidence of surgical site infection having surgical wound with pus discharge, serous or seropurulent discharge, signs of sepsis and diagnosed for surgical site infection from general surgical and orthopedic wards were enrolled. These patients were hospitalized for various period and 25(71\%) of them were treated with ceftriaxone and others were treated with antimicrobials such as metronidazole, vancomycin, ceftazidime, cloxacillin and gentamicin whereas there was no information on recent history of antimicrobial therapy for $5(14.3 \%)$ of the patients. In addition, patients with acute and chronic otitis media with clinically proven discharge from ENT outpatient ward were randomly recruited. Majority of these patients $34(61 \%)$ had history of recent treatment with 
ciprofloxacin and hydrogen peroxide, 4(6.8\%) with amoxicillin+clavulanic acid while the rest 19(32.2\%) had no history of recent antimicrobial therapy. Only patients who were willing to give their consent to participate in the study were involved.

\section{Sample collection and isolation of $S$. aureus}

A total of paired swab samples from 94 patients; wound swab from those with surgical site infection $(n=14$ from general surgical ward; $n=21$ from orthopedic ward) and ear swab from patients with otitis media having clinical symptom of ear discharge $(n=59)$ and corresponding nasal swabs from all patients $(n=94)$ were collected. The wound site and ear were first cleaned with sterile saline to remove any purulent debris. Sterile cotton swab was moistened with normal saline and rotated three times on the wound surface and ear opening and placed in test tubes containing $10 \mathrm{ml}$ of sterile Trypton Soya Broth(TSB), (BD, Diagnostic Systems, Heidelberg, Germany). Samples were transported in the ice box to the Microbiology Laboratory of Aklilu Lemma Institute of Pathobiology, Addis Ababa University within 3-4 h of collection and were immediately incubated at $37{ }^{\circ} \mathrm{C}$ overnight.

After overnight growth in TSB, loopful of the suspension was streaked into mannitol salt agar (Oxoid, Basingstoke, Hampshire, England). Then the plates were incubated at $37{ }^{\circ} \mathrm{C}$ for $24 \mathrm{~h}$ and bacterial colonies with typical characteristics of $S$. aureus (i.e. colonies with golden yellow pigmentation on mannitol salt agar) were subjected to subsequent biochemical tests involving Gram stain, catalase, and coagulase tests for confirmation. Staphylococcus aureus ATCC25923 was used as a reference strain.

\section{Antimicrobial susceptibility testing}

Antimicrobial susceptibility test for S. aureus was carried out against a panel of 17 antimicrobials using Kirby Bauer disc diffusion method according to Clinical and Laboratory Standards Institute guidelines [25] on Mueller Hinton agar (MHA) (Oxoid, Basingstoke, England). The bacterial culture was grown in TSB for $4-5 \mathrm{~h}$ at $37{ }^{\circ} \mathrm{C}$ and the inoculum density was adjusted with 0.5 McFarland standard. A sterile cotton swab was dipped into the suspension and it was pressed against the sides of the tube to avoid excess inoculum. The inoculum was evenly spread on MHA plate and kept for 15 min before antimicrobial discs were dispensed. The plates were then incubated at $37^{\circ} \mathrm{C}$ for $24 \mathrm{~h}$ and the diameter of zone of inhibition was measured using plastic transparent ruler. The interpretation of the categories of susceptible, intermediate or resistant was based on the CLSI guidelines [25]. Reference strain of $S$. aureus ATCC25923 was used as a quality control organism. The following antimicrobials with disk potencies $(\mu \mathrm{g})$ were used (SensiDiscs, Becton, Dickinson and Company, sparks, MD): oxacillin (Ox; 1), cefoxitin (Fox;30), cephalothin (Cf; 30), bacitracin (B;10 IU), clindamycin (Da; 2), ampicillin (Amp; $10 \mu \mathrm{g}$ ), amoxicillin-clavulanic acid (Amc; 30), ceftriaxone (Cro; 30), chloramphenicol (C) $(30 \mu \mathrm{g})$, ciprofloxacin (Cip;5), erythromycin (E;15), gentamicin ( $\mathrm{Gm}$; 10), amikacin (An; 30), sulphamethoxazole-trimethoprim (Sxt;25), doxycycline (Do,30), tetracycline (Te; 30), and nitrofurantoin(Nitro;300).

\section{Data analysis}

The chi-square test was employed to investigate association of sex and age of patients with carriage rate of $S$. aureus and MRSA. One way analysis of variance and student $\mathrm{t}$-test were used to compare the difference in the level of multidrug resistance in S. aureus originating from various wards and specimens. The difference between the means was considered significant at $p<0.05$.

\section{Results}

\section{Staphylococcus aureus infection rate}

Of all 188 specimens cultured for S. aureus, 79 (42.02\%) were positive. S. aureus was detected from 54 (57.4\%) of the 94 patients examined either from wound/ear discharge swab or nasal swab. Rate of recovery of $S$. aureus was higher among specimens obtained from patients with otitis media (44.1\% from ear discharge and 50.9\% from nasal swab) compared to wound and nasal swab of patients with surgical site infection which ranged from 28 to $38 \%$. However, overall S. aureus carriage rate per patient was almost similar among patients from various wards (Table 1). There was no statistically significant difference in occurrence of $S$. aureus among different age group and sex (Table 2).

\section{Antimicrobial resistance profile of $S$. aureus}

All $S$. aureus isolates examined in the current study were resistant to at least one of the 17 antimicrobials. All (100\%) of the isolates were resistant to ampicillin, 54(68.4\%) were resistant to oxacillin and cefoxitin, 50(63.3\%) to clindanmycin, $47(59.5 \%)$ to cephalothin, $45(57 \%)$ to tetracycline, 42(53.2\%) to sulphamethoxazole+trimethoprim, and bacitracin, 41(51.9\%) (Fig. 1).

Based on the sensitivity of isolates to cefoxitin and oxacillin, $54(68.4 \%)$ of the isolates were MRSA. There was no difference in the level of detection of MRSA among $S$. aureus isolates cultured from patients in different age group and from both sexes. Among isolates from various sources, MRSA was detected more frequently in isolates obtained from patients with surgical site infection $(p=0.001)$. Interestingly, all of the $23(100 \%)$ of isolates from general surgical and orthopedic wards were 
Table 1 Relative isolation rate of S. aureus from wound and nasal swab of patients with surgical site infection and ear discharge swab and nasal swab of patients with otitis media

\begin{tabular}{|c|c|c|c|c|c|c|}
\hline Patient category & No. of patients & $\begin{array}{l}\text { No. of patients positive } \\
\text { from one or more specimen (\%) }\end{array}$ & Specimen & No. examined & No. (\%) positive & Total no. of isolates \\
\hline \multirow[t]{2}{*}{ General surgical ward } & 14 & $8(57.1)$ & Wound swab & 14 & $4(28.6)$ & 8 \\
\hline & & & Nasal swab & 14 & $4(28.6)$ & \\
\hline \multirow[t]{2}{*}{ Orthopedic ward } & 21 & $10(47.6)$ & Wound swab & 21 & $8(38.1)$ & 15 \\
\hline & & & Nasal swab & 21 & $7(33.3)$ & \\
\hline \multirow[t]{2}{*}{ ENT ward } & 59 & $36(61)$ & Ear discharge & 59 & $26(44.1)$ & 56 \\
\hline & & & Nasal swab & 59 & $30(50.9)$ & \\
\hline Total & 94 & $54(57.5)$ & & 188 & $79(42.02)$ & 79 \\
\hline
\end{tabular}

MRSA whereas only 31(54.4\%) of isolates obtained from patients with ear infection were MRSA (Table 3).

Resistance to two or more antimicrobials was recorded in $74(95 \%)$ of the isolates, while resistance to 3 or more antimicrobials was detected in $65(82.3 \%)$ of the isolates. Multidrug resistance to 7 or more antimicrobials was detected in 51(64.6\%) of the isolates. Majority of isolates cultured from surgical site infection were resistant to several drugs. One of the isolate cultured from a wound swab of patient with surgical site infection from orthopedic ward was found to be resistant to all of the 17 antimicrobials tested (Table 4).

The mean $( \pm$ SEM) number of drugs to which $S$. aureus isolates obtained from patients from ENT, general surgical ward and orthopedic ward were 7.1 $\pm 0.6,10.63$ $\pm 1.2,13.3 \pm 0.7$, respectively (Fig. $2 \mathrm{a}$ ). The level of MDR was significantly higher in $S$. aureus isolates obtained from patients in orthopedic ward compared to those obtained from general surgical ward as well as ENT wards $(p<0.0001)$ while no significant difference was observed among isolates obtained from other two wards. Similarly, comparison of level of MDR among S. aureus strains isolated from various specimens revealed that strains isolated from wound swab were resistant to significantly

Table 2 Sociodemographic characteristics of patients with surgical site infection, ear infection and carriage rate of S. aureus at Tikur Anbessa Specialized Hospital

\begin{tabular}{lllll}
\hline Characteristics & $\begin{array}{l}\text { No. (\%) } \\
\text { tested }\end{array}$ & $\begin{array}{l}\text { No. (\%) S. aureus } \\
\text { culture positive }\end{array}$ & $X^{2}$ & $p$-value \\
\hline Sex & & & & \\
$\quad$ Male & 54 & $28(51.8)$ & 1.6 & 0.29 \\
$\quad$ Female & 40 & $26(65)$ & & \\
Age in years & & & & \\
$<11$ & 18 & $9(50)$ & 3.87 & 0.42 \\
$11-20$ & 26 & $19(73.1)$ & & \\
$21-30$ & 20 & $11(55)$ & & \\
$31-40$ & 15 & $8(53.3)$ & & \\
$\geq 41$ & 15 & $7(46.7)$ & & \\
\hline
\end{tabular}

larger number of antimicrobials compared to those isolated from nasal swab as well as ear swab $(p<0.001)$ (Fig. 2b).

\section{Discussion}

The objective of the current study was to assess occurrence and antimicrobial resistance profile of $S$. aureus among patients with surgical site infection and ear infection and corresponding nasal swab at Tikur Anbessa Specialized Hospital. The overall rate of recovery of $S$. aureus among patients with surgical site infection in the current study (47.6-57.1\%) is in line with previous studies in which $S$. aureus was shown to be the predominant pathogen responsible for surgical site infection in Ethiopia [6]. Similarly high rate of isolation of S. aureus was reported from patients with pus/wound discharge at Gondar University hospital in north Ethiopia [26], and from ear discharges from Hawassa Hospital, southern Ethiopia [27]. In some of the patients with surgical site infection in the current study, S. aureus was not detected whereas the same patients were positive from nasal swab. Such heterogeneity could be due to direct topical application of antimicrobials to the infection site which might have affected growth of bacteria from this site. The other possible reason could be the real absence of $S$. aureus from infection site and detection from nasal swab might be due to natural colonization [1]. On the other hand, the absence of $S$. aureus from nasal swab and detection from wound swab and ear discharge could be due to localized infection in the specific infected sites.

Detection of S. aureus from ear discharge of $44.1 \%$ of patients with otitis media in the current study shows that $S$. aureus is one of the major causes of ear infection in the study population. In previous studies in Ethiopia, $S$. aureus was the dominant bacterial pathogen isolated from patients with otitis media in Mekelle, north Ethiopia [8] and the 2nd predominantly isolated pathogen in northeastern Ethiopia [28]. Unlike previous studies where recovery of bacterial pathogens including $S$. aureus varied among patients with different age group $[8,28]$, in the current study there was no significant 


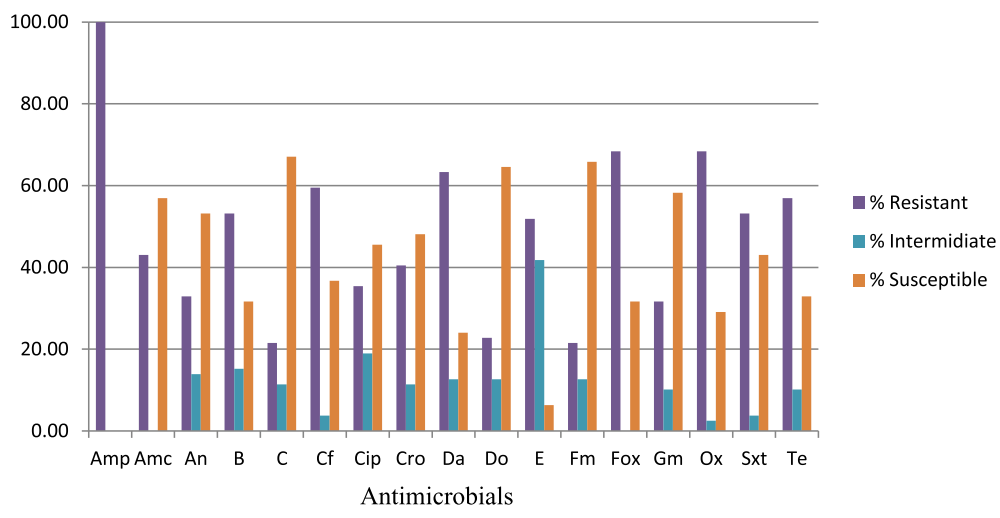

Fig. 1 Proportion of susceptible, intermediately resistant and resistant S. aureus isolates $(n=79)$ to 17 antimicrobials examined $(A m p=$ ampicillin; Amc = amoxicillin+clavulanic acid; $A n=$ amikacin; $B=$ bacitracin; $C=$ chloramphenicol; $C f=$ cephalothin; $C i p=$ ciprofloxacin; $C r o=$ ceftriaxone; Fox = cefoxitin; $\mathrm{Da}=$ clindamycin Do = doxycycline; $\mathrm{E}=$ erythromycin; $\mathrm{Gm}=$ gentamicin; Fm = nitrofurantoin; Ox = oxacillin, Sxt = sulfamethoxazole + trimethoprim; Te = tetracycline)

difference in isolation rate of $S$. aureus among age groups, which could be due to small sample size in the current study and due to difference in composition of patient category.

The current study also revealed high rate of resistance to most of the antimicrobials by strains of $S$. aureus isolated from patients with surgical site infection and those with otitis media. Of particular concern is that all strains in this study were resistant to ampicillin unlike previous studies where resistance to ampicillin was observed in 82.2 and $88.5 \%$ of $S$. aureus isolates from patients with surgical site infection at Debre Markos Referral Hospital, northwest Ethiopia [29], and patients with otitis media in northeast Ethiopia [28], respectively. On the other hand, it is in agreement with the finding where $100 \%$ resistance to ampicillin was recorded in S. aureus strains

Table 3 Relative distribution of MRSA among S. aureus isolates from patients of different sex, age group and ward type

\begin{tabular}{lllll}
\hline $\begin{array}{l}\text { Characteristics of } \\
\text { source of isolates }\end{array}$ & No. S. aureus & No. MRSA (\%) & $X^{2}$ & $p=$ value \\
\hline Sex & & & & \\
$\quad$ Male & 42 & $28(66.7)$ & 0.12 & 0.87 \\
$\quad$ Female & 37 & $26(70.3)$ & & \\
Age in years & & & & \\
$<11$ & 16 & $12(75)$ & & \\
$11-20$ & 25 & $16(64)$ & 2.5 & 0.65 \\
$21-30$ & 18 & $12(66.7)$ & & \\
$31-40$ & 12 & $7(58.3)$ & & \\
$\geq 41$ & 8 & $7(87.5)$ & & \\
Ward type & & & & \\
General surgical & 8 & $8(100)$ & 15.02 & 0.001 \\
Orthopedic & 15 & $15(100)$ & & \\
ENT & 56 & $31(54.4)$ & & \\
\hline
\end{tabular}

isolated from patients admitted to Felege Hiwot referral Hospital, North Ethiopia [6]. Such difference could be attributed to variation in patient hospital stay, level of infection control practices by health facilities, and previous exposure of patient to antimicrobials [30]. Some isolates from nasal swab and respective wound or ear discharge swab from a single patient had different antimicrobial susceptibility profile. The possible reason for this could be due to localized infection with different strain of $S$. aureus at the wound site while the one from nasal swab could be due to natural colonization.

Rate of occurrence of MRSA (68.4\%) out of total $S$. aureus infection in the current study is higher than study reported by Kahsay et al. [29] from patients with surgical site infection at Debre Markos Referral Hospital, northern Ethiopia (49.7\%), various clinical specimens from Yekatit 12 Hospital, in Addis Ababa which was 17.5\% [24] as well as reports from HIV infected pediatric patients in northwest Ethiopia [31]. It is also higher than a study in Philippines where $S$. aureus isolated from clinical specimens showed 40\% MRSA [32]. This could be attributed to difference in composition of patients from whom samples were collected including patients being outpatients, hospitalized, duration of hospital stay and previous antimicrobial use. Majority of MRSA strains in the current study were isolated from hospitalized patients with surgical site infections and those with chronic otitis media who received antimicrobials. Interestingly, all isolates obtained from patients from general surgical and orthopedic wards were MRSA strains, unlike those from ENT ward where only $54 \%$ were MRSA suggesting higher rate of infection of patients with MDR strains from the hospital environment in hospitalized patients either during surgical procedure or during postoperative care. Previous study indicated that most of surgical site infections occur during surgery 
Table 4 Resistance pattern of S. aureus strains isolated from patients from different wards and specimens

\begin{tabular}{|c|c|c|c|c|}
\hline Resistance pattern & $\begin{array}{l}\text { No. of drugs to } \\
\text { which } R \text { is observed }\end{array}$ & $\begin{array}{l}\text { No. (\%) } \\
\text { isolates }\end{array}$ & Ward type & Specimen \\
\hline Amp & 1 & $4(5.1)$ & ENT & Nasal swab(2)Ear swab(2) \\
\hline $\begin{array}{l}\text { AmpSxt(2),AmpDa,AmpDo, AmpE(2), } \\
\text { AmpFox(2), AmpTe(2) }\end{array}$ & 2 & $10(12.7)$ & ENT & Ear swab(5), Nasal swab(5) \\
\hline AmpBSxt, AmpDaOx, AmpTeSxt & 3 & 3(3.8) & ENT & Ear swab(1), Nasal swab(2) \\
\hline $\begin{array}{l}\text { AmpCfFoxOx, AmpDaDoTe,AmpFoxOxSxt, } \\
\text { AmpBCfDa }\end{array}$ & 4 & $4(5.1)$ & ENT & Ear swab(2) nasal swab(2) \\
\hline $\begin{array}{l}\text { AmpBDoFoxTe, AmpBEOxSxt, AmpDaESxtTe, } \\
\text { AmpDoFoxTeOx, AmpBDaETe }\end{array}$ & 5 & $5(6.3)$ & ENT(4), Surgical(1) & Ear swab(2), nasal swab(3) \\
\hline AmpBDaFoxSxtTe, AmpCEFoxOxTe & 6 & $2(2.5)$ & ENT & Ear swab(1), nasal swab(1) \\
\hline $\begin{array}{l}\text { AmpAnCDaEFoxTe, AmpAnDaFoxOxSxtTe, } \\
\text { AmpBCfDaFmFoxOx, AmpBCfCroDaFoxOx, } \\
\text { AmpBCfDaFmFoxSxt }\end{array}$ & 7 & $5(6.3)$ & ENT(4), Surgical(1) & Ear swab(2), nasal swab(3) \\
\hline $\begin{array}{l}\text { AmpAnCfCroFoxGmOxTe, AmpAnDaDoFoxGmOxTe, } \\
\text { AmpBCfDaDoFoxOxTe, AmpBCfDaEOxSxtTe, } \\
\text { AmpBCipCroFmGmOxSxt }\end{array}$ & 8 & $5(6.3)$ & ENT(2), Surgical(1), Ortho(2), & $\begin{array}{l}\text { Nasal swab(1), wound } \\
\operatorname{swab}(2) \text {, ear swab(2), }\end{array}$ \\
\hline $\begin{array}{l}\text { AmpAnCfDaFoxGmOxSxtTe AmpCCfCipCroDaFoxETe, } \\
\text { AmpCCfCipCroFoxGmOx } \\
\text { AmpAnBCipDaFoxOxSxtTe, AmpBCCfCroDaFoxGmOx, } \\
\text { AmpBCCfCroFoxOxSxtTe }\end{array}$ & 9 & $6(7.6)$ & ENT (5), Ortho(1) & Nasal swab(5), ear swab (1) \\
\hline $\begin{array}{l}\text { AmpBCCfCipDaEFmFoxOx, } \\
\text { AmpBCCfCipDaEOxSxtTe } \\
\text { AmpBCCCCroDaFmFoxOxSxt, AmpBCCfDaDoEFmOxTe }\end{array}$ & 10 & $4(5.1)$ & ENT(3), Surgical(1) & Ear swab(3), Wound swab(1) \\
\hline $\begin{array}{l}\text { AmpAnBCfCipDaDoEFoxOxTe, } \\
\text { AmpAnBCCfCroDaDoFoxOxTe, } \\
\text { AmpAnCCipCroDaEFoxOxSxtTe, } \\
\text { AmpAnCCipCroDaEFoxOxSxtTe, } \\
\text { AmpAnCCfCipCroDaEFoxGmOx, } \\
\text { AmpAmcAnCCfCipEFoxOxSxtTe, } \\
\text { AmpAmcBCfCroDaEFmFoxOxSxt, } \\
\text { AmpAmcAnBCfCroDaEFmFoxOx, } \\
\text { AmpAmcBCfCipCroEFoxOxSxtTe, } \\
\text { AmpAnBCfCroDaEFoxGmOxTe }\end{array}$ & 11 & $9(11.4)$ & ENT(6), Ortho(2), Surgical(1) & $\begin{array}{l}\text { Ear swab(2), nasal swab(6), } \\
\text { wound swab(1) }\end{array}$ \\
\hline $\begin{array}{l}\text { AmpAnBCfDaDoEFoxGmOxSxtTe, } \\
\text { AmpAmcAnBCfDaEFmFoxOxSxtOx, } \\
\text { AmpAmcAnBCCfCroDaEFoxOxTe, } \\
\text { AmpAmcBCfCipCroEFoxGmOxSxte, } \\
\text { AmpAmcBCCCipDaDoEFmOxSxtTe }\end{array}$ & 12 & $5(6.3)$ & ENT(3), Ortho(2) & $\begin{array}{l}\text { Ear swab(2), nasal swab(1), } \\
\text { Wound swab(2) }\end{array}$ \\
\hline $\begin{array}{l}\text { AmpAmcAnCCfCipCroDaEFoxGmOxSxt, } \\
\text { AmpAmcBCfCipCroDaEFoxGmOxSxtTe, } \\
\text { AmpAmcAnBCfCipCroEFmFoxGmOxSxt, } \\
\text { AmpCCfCipCroDaDoEFoxGmOxSxtTe, } \\
\text { AmpAnCCfCipCroDaDoFoxGmOxSxtTe, } \\
\text { AmpAmcBCCfCipCroDaFoxGmOxSxtTe, } \\
\text { AmpAmcBCfCipCroDaFmFoxGmOxSxtTe }\end{array}$ & 13 & $7(8.9)$ & ENT(2), Surgical(2), Ortho(3) & $\begin{array}{l}\text { Ear swab(2), wound } \\
\text { swab(2), nasal swab(3) }\end{array}$ \\
\hline $\begin{array}{l}\text { AmpAmcAnCCfCipCroDaEFoxGmOxSxtTe, } \\
\text { AmpAmcAnBCfCipCroDaEFoxGmOxSxtTe, } \\
\text { AmpAmcAnCfCipCroDaDoEFoxGmOxSxtTe, } \\
\text { AmpAmcAnBCfCipCroDaEFoxGmOxSxtTe, } \\
\text { AmpAmcAnBCCfCipCroDaEFoxOxSxtTe, }\end{array}$ & 14 & $5(6.3)$ & Ortho(3), ENT(2) & Nasal swab(4), wound swab(1) \\
\hline $\begin{array}{l}\text { AmpAmcBCCfCipCroDaEFmFoxGmOxSxtTe, } \\
\text { AmpAmcAnBCCfCipCroDaEFoxGmOxSxtTe, } \\
\text { AmpAmcBCCfCipCroDaDoEFoxGmOxSxtTe }\end{array}$ & 15 & 3(3.8) & Surgical(1) Ortho(2) & Wound swab (2), nasal swab(1) \\
\hline AmpAmcAnBCCfCipCroDaDoEFoxGmOxSxtTe & 16 & $1(1.3)$ & $\operatorname{EVNT}(1)$ & Ear swab(1) \\
\hline AmpAmcAnBCCfCipCroDaDoEFmFoxGmOxSxtTe & 17 & $1(1.3)$ & Ortho & Wound swab(1) \\
\hline
\end{tabular}

Amp ampicillin, Amc amoxicillin+clavulanic acid, An amikacin, B bacitracin, Cf cephalothin, C chloramphenicol, Cip ciprofloxacin, Cro ceftriaxone, Fox cefoxitin, Da clindamycin, Do doxycycline, E erythromycin, Gm gentamicin, Nitro nitrofurantoin, Ox oxacillin, Sxt sulfamethoxazole + trimethoprim, $T e$ tetracycline, $R$ resistant 

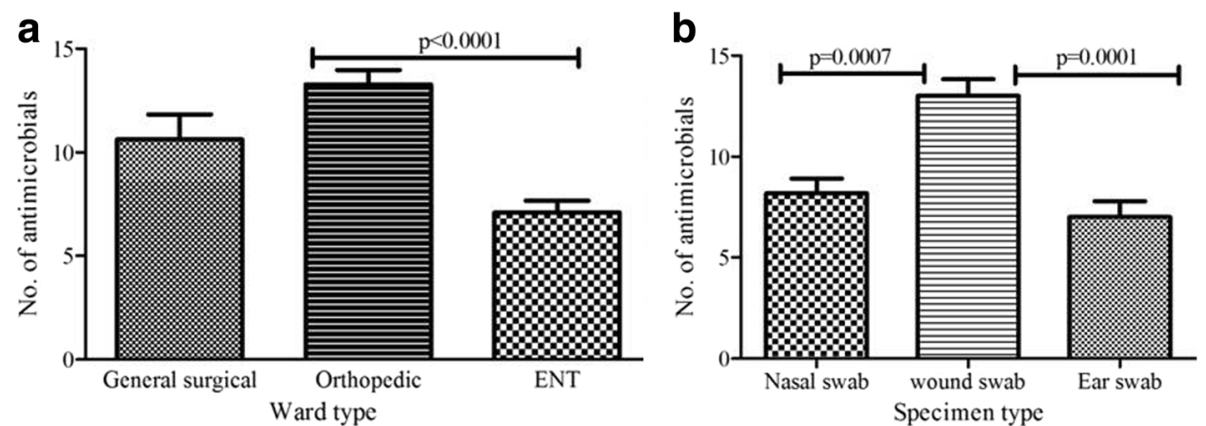

Fig. 2 Level of multi-drug resistance among S. aureus isolates from different wards and different specimens (Mean number of antimicrobials to which isolates were resistant were compared $\mathbf{a}$, Irrespective of specimen type, mean number of drugs to which isolates obtained from orthopedic ward were resistant was significantly higher than those from ENT wards $(p<0.0001) \mathbf{b}$, Strains of $S$. aureus isolated from wound swab were MDR to several drugs compared to those obtained from nasal swab $(p=0.0007)$ and ear swab $(p=0.0001)$

demonstrated by matching of strains of pathogens from surgeon's fingers and post-operative infection [33].

The probable reason for high rate of overall MDR in $S$. aureus isolates obtained from orthopedic surgery ward compared to those from other wards could be due to high contamination of this specific ward with MDR strains of $S$. aureus. The reason why isolates cultured from nasal swab and ear swab were resistant to less number of drugs compared to those obtained from surgical wound could possibly because the level of previous exposure to antimicrobial agents might not be as high as those from surgical ward who were hospitalized. All isolates from ear discharge and most of the isolates from nasal swab were obtained from outpatients in ENT ward who had less exposure to antimicrobials. MRSA and other MDR nosocomial pathogens can be transmitted through skin contact among patients, from health personnel and hospital environment and surfaces unless proper infection control measures are practiced [34]. The poor sanitary conditions of the hospital and lack of routine surveillance of antimicrobial susceptibility of circulating bacterial strains might have contributed to spread of resistant bacteria in the hospital.

\section{Conclusion}

In general, this study revealed high rate of MDR strains of $S$. aureus in TASH, majority of which being MRSA. As this study involved limited wards in a single hospital and small sample size, it may not represent the whole hospital situation and the country as well. Despite this limitation, the finding warrants implementation of measures to control spread of MDR strains in the hospital and the community. Some of the measures to be undertaken include, improving antimicrobial stewardship through routine monitoring of antimicrobial susceptibility of circulating strains and avoiding or reducing empirical therapy, effective infection control practices, training of health personnel and patients on the risk of antimicrobial resistance.

\section{Abbreviations}

CLSI: Clinical and Laboratory Standards Institute; ENT: Ear nose and throat: MDR: multidrug resistance; MHA: Mueller Hinton agar; MRSA: Methicillin resistant Staphylococcus aureus; TASH: Tikur Anbessa Specialized Hospital; TSB: Trypton Soya Broth

\section{Acknowledgements}

The authors would like to thank staff of ENT, surgical and orthopedic wards of TASH for their technical support during sample collection and all study participants.

\section{Funding}

This study was financially supported by Addis Ababa University.

\section{Availability of data and materials}

All data are included in this published article and all raw data can be available upon request from the corresponding author.

\section{Authors' contributions}

ST, TE, AT, GT, TS and WS participated in conception of the study. ST was involved in sample collection, laboratory work and preparation of draft manuscript. TE supervised data collection and participated in preparation of draft manuscript. HA participated in laboratory investigation. All authors read and approved the final manuscript.

\section{Ethics approval and consent to participate}

Study protocol was approved by the Ethical Review Board of School of Pharmacy, College of Health Sciences, Addis Ababa University. Individual verbal informed consent was obtained from all adult participants and the parents or guardians of all children who participated in the study following adequate explanation about the purpose of the study.

\section{Competing interests}

The authors declare that they have no competing interests.

\section{Publisher's Note}

Springer Nature remains neutral with regard to jurisdictional claims in published maps and institutional affiliations.

\section{Author details}

${ }^{1}$ Yekatit 12 Hospital Medical College, P.O. Box157, Addis Ababa, Ethiopia. ${ }^{2}$ Aklilu Lemma Institute of Pathobiology, Addis Ababa University, P.O. Box 1176, Addis Ababa, Ethiopia. ${ }^{3}$ Department of Internal Medicine, School of Medicine, College of Health Sciences, Addis Ababa University, Churchill Avenue, P.O.Box 9086, Addis Ababa, Ethiopia. ${ }^{4}$ Department of Biomedical Sciences, College of Veterinary medicine and Agriculture, Addis Ababa 
University, P.O. Box 34, Debrezeit, Ethiopia. ${ }^{5}$ Institute of Biotechnology, College of natural and Computational sciences, Addis Ababa University, P.O. Box, 1176, Addis Ababa, Ethiopia. ${ }^{6}$ Department of Pharmacology and Clinical Pharmacy, School of Pharmacy, College of Health Sciences, Addis Ababa University, Churchill Avenue, P.O. Box 9086, Addis Ababa, Ethiopia.

Received: 18 September 2017 Accepted: 26 April 2018

Published online: 21 May 2018

\section{References}

1. Wertheim HF, Melles DC, Vos MC, van Leeuwen W, van Belkum A, Verbrugh HA, Nouwen $J$ L. The role of nasal carriage in Staphylococcus aureus infections. Lancet Infect Dis. 2005;5(12):751-62.

2. Tong SY, Davis JS, Eichenberger E, Holland TL, Fowler VG Jr. Staphylococcus aureus infections: epidemiology, pathophysiology, clinical manifestations, and management. Clin Microbiol Rev. 2015;28(3):603-61.

3. Davies J, Davies D. Origins and evolution of antibiotic resistance. Microbiol Mol Biol Rev. 2010;74(3):417-33.

4. Mulvey MR, Simor AE. Antimicrobial resistance in hospitals: how concerned should we be? CMAJ. 2009;180(4):408-15.

5. Cheadle WG. Risk factors for surgical site infection. Surg Infect. 2006;7(Suppl 1):S7-11.

6. Mulu W, Kibru G, Beyene G, Damtie M. Postoperative nosocomial infections and antimicrobial resistance pattern of Bacteria isolates among patients admitted at Felege Hiwot referral hospital, Bahirdar, Ethiopia. Ethiop J Health Sci. 2012:22(1):7-18.

7. Abera B, Biadeglegne F. Antimicrobial resistance patterns of Staphylococcus aureus and Proteus spp. isolated from otitis media at Bahir Dar regional laboratory, north West Ethiopia. Ethiop Med J. 2009;47(4):271-6.

8. Wasihun AG, Zemene Y. Bacterial profile and antimicrobial susceptibility patterns of otitis media in Ayder Teaching and Referral Hospital, Mekelle University, Northern Ethiopia. Springerplus. 2015;4:701. https://doi.org/10. 1186/s40064-015-1471-z

9. Kesah C, Ben Redjeb S, Odugbemi TO, Boye CS, Dosso M, Ndinya Achola JO, Koulla-Shiro S, Benbachir M, Rahal K, Borg M. Prevalence of methicillinresistant Staphylococcus aureus in eight African hospitals and Malta. Clin Microbiol Infect. 2003;9(2):153-6.

10. Rauber JM, Carneiro M, Arnhold GH, Zanotto MB, Wappler PR, Baggiotto B, Valim AR, d'Azevedo PA. Multidrug-resistant Staphylococcus spp and its impact on patient outcome. Am J Infect Control. 2016;44(11):e261-3.

11. Katayama Y, Ito T, Hiramatsu K. A new class of genetic element, staphylococcus cassette chromosome mec, encodes methicillin resistance in Staphylococcus aureus. Antimicrob Agents Chemother. 2000:44(6):1549-55.

12. Garcia-Alvarez L, Holden MT, Lindsay H, Webb CR, Brown DF, Curran MD, Walpole E, Brooks K, Pickard DJ, Teale C, et al. Meticillin-resistant Staphylococcus aureus with a novel mecA homologue in human and bovine populations in the UK and Denmark: a descriptive study. Lancet Infect Dis. 2011;11(8):595-603.

13. Bhattacharya S, Pal K, Jain S, Chatterjee SS, Konar J. Surgical site infection by methicillin resistant Staphylococcus aureus- on decline? J Clin Diagn Res. 2016;10(9):DC32-6.

14. Stefani S, Chung DR, Lindsay JA, Friedrich AW, Kearns AM, Westh $H$, Mackenzie FM. Meticillin-resistant Staphylococcus aureus (MRSA): global epidemiology and harmonisation of typing methods. Int J Antimicrob Agents. 2012;39(4):273-82.

15. Nwankwo EO, Nasiru MS. Antibiotic sensitivity pattern of Staphylococcus aureus from clinical isolates in a tertiary health institution in Kano, Northwestern Nigeria. Pan Afr Med J. 2011:8:4.

16. Takano T, Higuchi W, Zaraket H, Otsuka T, Baranovich T, Enany S, Saito K, Isobe H, Dohmae S, Ozaki K, et al. Novel characteristics of communityacquired methicillin-resistant Staphylococcus aureus strains belonging to multilocus sequence type 59 in Taiwan. Antimicrob Agents Chemother. 2008;52(3):837-45

17. Aires-de-Sousa M. Methicillin resistant Staphylococcus aureus among animals:current overview. Clin Microbiol Infect. 2016;23(6):373-80.

18. Guimares FF, Manzi MP, Joaquim SF, Richini-Pereira VB, Langoni H. Outbreak of methicillin-resistant Staphylococcus aureus (MRSA)-associated mastitis in a closed dairy herd. J Dairy Sci. 2017;100(1):726-30.

19. Holden MT, Feil EJ, Lindsay JA, Peacock SJ, Day NP, Enright MC, Foster TJ, Moore CE, Hurst L, Atkin R, et al. Complete genomes of two clinical
Staphylococcus aureus strains: evidence for the rapid evolution of virulence and drug resistance. Proc Natl Acad Sci U S A. 2004;101(26):9786-91.

20. Cameron DR, Howden BP, Peleg AY. The interface between antibiotic resistance and virulence in Staphylococcus aureus and its impact upon clinical outcomes. Clin Infect Dis. 2011;53(6):576-82.

21. Morgan DJ, Okeke IN, Laxminarayan R, Perencevich EN, Weisenberg S. Nonprescription antimicrobial use worldwide: a systematic review. Lancet Infect Dis. 2011;11(9):692-701.

22. Vernet G, Mary C, Altmann DM, Doumbo O, Morpeth S, Bhutta ZA, Klugman KP. Surveillance for antimicrobial drug resistance in under-resourced countries. Emerg Infect Dis. 2014;20(3):434-41.

23. Okeke IN, Lamikanra A, Edelman R. Socioeconomic and behavioral factors leading to acquired bacterial resistance to antibiotics in developing countries. Emerg Infect Dis. 1999;5(1):18-27.

24. Dilnessa T, Bitew A. Prevalence and antimicrobial susceptibility pattern of methicillin resistant Staphylococcus aureus isolated from clinical samples at Yekatit 12 hospital medical college, Addis Ababa, Ethiopia. BMC Infect Dis. 2016;16:398.

25. CLSI. Performance standards for antimicrobial susceptibility testing; twentythird informational SupplementM100-S23, vol. 33; 2013.

26. Muluye D, Wondimeneh $Y$, Ferede $G$, Nega T, Adane $K$, Biadgo B, Tesfa H, Moges F. Bacterial isolates and their antibiotic susceptibility patterns among patients with pus and/or wound discharge at Gondar university hospital. BMC Res Notes. 2014;7:619.

27. Deyno S, Toma A, Worku M, Bekele M. Antimicrobial resistance profile of staphylococcus aureus isolates isolated from ear discharges of patients at University of Hawassa comprehensive specialized hospital. BMC Pharmacol Toxicol. 2017;18(1):35.

28. Argaw-Denboba A, Abejew AA, Mekonnen AG. Antibiotic-resistant Bacteria are major threats of otitis Media in Wollo Area, northeastern Ethiopia: a tenyear retrospective analysis. Int J Microbiol. 2016;2016:8724671.

29. Kahsay A, Mihret A, Abebe T, Andualem T. Isolation and antimicrobial susceptibility pattern of Staphylococcus aureus in patients with surgical site infection at Debre Markos referral hospital, Amhara region, Ethiopia. Arch Public Health. 2014;72(1):16.

30. Harrop JS, Styliaras JC, Ooi YC, Radcliff KE, Vaccaro AR, Wu C. Contributing factors to surgical site infections. J Am Acad Orthop Surg. 2012;20(2):94101.

31. Lemma MT, Zenebe Y, Tulu B, Mekonnen D, Mekonnen Z. Methicillin resistant Staphylococcus aureus among HIV infected pediatric patients in Northwest Ethiopia: carriage rates and antibiotic co-resistance profiles. PLOS One. 2015;10(9):e0137254

32. Juayang AC, de Los Reyes GB, de la Rama AJ, Gallega CT. Antibiotic resistance profiling of Staphylococcus aureus isolated from clinical specimens in a tertiary hospital from 2010 to 2012. Interdiscip Perspect Infect Dis. 2014;2014:898457.

33. Uckay I, Hoffmeyer P, Lew D, Pittet D. Prevention of surgical site infections in orthopaedic surgery and bone trauma: state-of-the-art update. J Hosp Infect. 2013;84(1):5-12.

34. Wang $X$, Xiao Y, Wang J, Lu X. A mathematical model of effects of environmental contamination and presence of volunteers on hospital infections in China. J Theor Biol. 2012;293:161-73.

\section{Ready to submit your research? Choose BMC and benefit from:}

- fast, convenient online submission

- thorough peer review by experienced researchers in your field

- rapid publication on acceptance

- support for research data, including large and complex data types

- gold Open Access which fosters wider collaboration and increased citations

- maximum visibility for your research: over $100 \mathrm{M}$ website views per year

At BMC, research is always in progress.

Learn more biomedcentral.com/submissions 